\title{
Messen ohne Maß? : Nicolaus Cusanus und das Kriterium menschlicher Erkenntnis
}

\section{Kny, Christian}

2018

Kny , C 2018 , ' Messen ohne Maß? Nicolaus Cusanus und das Kriterium menschlicher

Erkenntnis ' , Das Mittelalter , vol. 23 , no. 1 , pp. 92-108 . https://doi.org/10.1515/mial-2018-0007

http://hdl.handle.net/10138/313229

https://doi.org/10.1515/mial-2018-0007

publishedVersion

Downloaded from Helda, University of Helsinki institutional repository.

This is an electronic reprint of the original article.

This reprint may differ from the original in pagination and typographic detail.

Please cite the original version. 


\title{
Christian Kny* \\ Messen ohne Maß? Nicolaus Cusanus und das Kriterium menschlicher Erkenntnis
}

\author{
https://doi.org/10.1515/mial-2018-0007
}

\begin{abstract}
In the late Middle Ages, Nicholas of Cusa renders human cognition as creative, asymptotic assimilation-humans creatively approach their objects of cognition without ever fully reaching them. Questions about measuring are an important part of Nicholas' model of cognition in two regards: On the one hand, he explicitly calls human cognition a 'measuring' (mensurare), moving the concept into the centre of attention. On the other hand, measuring in the sense of evaluating epistemic activities is an issue for Nicholas. He describes humans as living images of god who 'enfold' (complicare) the ideas of all things within themselves in a specific way. They measure, i. e. judge, their epistemic activities looking at what they enfold. However, Nicholas provides little information about what exactly that means. He is thus threatened with a serious epistemological problem: the lack of a satisfying criterion of epistemic activities.

In my paper, I discuss options of how to deal with this problem. After briefly describing (1) Nicholas' notion of human cognition and (2) what he has on offer regarding a criterion of epistemic activities, I (3) try to clarify what 'enfolding the ideas of all things' can mean. Presenting and discussing two plausible interpretations of this expression-a static and a dynamic one-sheds light on possible answers Nicholas can give as well as the limitations these answers are confronted with.
\end{abstract}

Keywords: Nicholas of Cusa, epistemology, epistemic justification, iudicium concreatum, complicatio

\footnotetext{
Hinweis: Dieser Beitrag wurde vom European Research Council im Rahmen des Förderungsvertrags Nr. 637747 für das Project, Rationality in Perception: Transformations of Mind and Cognition 1250-1550` gefördert.

*Kontakt: Christian Kny, ERC StG project, Rationality in Perception: Transformations of Mind and Cognition 1250-1550, University of Helsinki, Department of Philosophy, History, Arts and Culture Studies PO Box $24 \mathrm{Fl}-00014$ Helsinki
} 
Die Frage nach Maß und Messen ist eine der Kernfragen jedes erkenntnistheoretischen Entwurfs. Sofern Erkennen nicht als ein Prozess gedacht wird, der notwendig erfolgreich verläuft, muss geklärt werden, wie Erkenntnisbemühungen auf ihre Korrektheit geprüft und so Erkenntnisansprüche validiert werden können. Zugespitzt: Ohne Maß, d.h. ohne Kriterium, ist Erkenntnis als methodisch abgesichertes Verfahren nicht möglich.

Nicolaus Cusanus konzipiert menschliche Erkenntnis im ausgehenden Mittelalter als kreative, asymptotische Assimilation - Menschen nähern sich auf kreative Weise ihren Erkenntnisobjekten an, können diese aber nicht gänzlich erreichen. Die Frage nach dem Maß ist mit Blick auf diese Konzeption in zweifacher Hinsicht bedeutsam: Einerseits bezeichnet Cusanus die Erkenntnistätigkeit von Menschen explizit als ein Messen (mensurare). Andererseits ist gerade die Frage nach dem Maß im Sinne eines Kriteriums menschlicher Erkenntnis bei Cusanus drängend. Er charakterisiert Menschen als lebendige Bilder Gottes, die als solche die Urbilder alles Seienden auf spezifisch menschliche Weise in sich tragen. Diese Urbilder sind für Menschen dasjenige, an dem sie ihr erkennendes Messen ausrichten. Im Blick darauf, wie die spezifisch menschliche Weise dieses In-SichTragens von Urbildern genau zu verstehen ist, hält sich Cusanus jedoch bedeckt. Das ist problematisch - ist bei ihm keine plausible Stellungnahme zur Kriterienfrage $\mathrm{zu}$ finden, so droht ihm buchstäblich erkenntnistheoretische Maßlosigkeit: Messen könnte von Nicht-Messen nicht unterschieden werden, wenn es kein Maß zur Prüfung von Erkenntnisbemühungen gibt.

Nach kurzen Erläuterungen (1) zum cusanischen Verständnis menschlicher Erkenntnis und (2) zur Kriterienfrage bildet (3) eine Auseinandersetzung mit den interpretatorischen Lösungsoptionen für dieses Problem deshalb den Kern des vorliegenden Beitrags. Ich vertrete dabei die These, dass Cusanus zwar einen in den Grundzügen tragfähigen Umgang mit der Kriterienfrage skizziert - er spricht Menschen eine anerschaffene Urteilsfähigkeit zu, die Erkenntnisvorgänge mit Blick auf das prüft, was Menschen als lebendige Bilder Gottes in sich tragen. Die Detailarmut dieser Skizze sorgt jedoch für interpretatorische Herausforderungen, die ich hier für zwei plausible Interpretationsstränge zwar ansprechen und zur Diskussion stellen, aus Umfangsgründen aber nicht erschöpfend behandeln kann.

Ebenfalls aus Umfangsgründen beschränke ich mich auf ,Idiota de mente، (kurz: ,De mente') als Textgrundlage. Das bietet sich aus drei Gründen an. Erstens liefert Cusanus dort eine ausgewogenere Darstellung menschlicher Erkenntnis als in anderen Werken. ${ }^{1}$ Zweitens macht die Charakterisierung des menschlichen

1 Chronologisch ungefähr in der Mitte von Cusanus' (philosophischer) Schaffenszeit angesiedelt, werden in ,De mente' unter ontologischen und epistemologischen Gesichtspunkten die Beschaf- 
Geistes als messende Instanz in ,De mente deutlich, was von Cusanus' Standpunkt aus epistemologisch unter ,messen` $z u$ verstehen ist. Drittens spricht Cusanus die Kriterienfrage in ,De mente‘ explizit an und legt damit auch mögliche Schwachpunkte offen.

\section{Menschliche Erkenntnis und die Kriterienfrage}

Menschliche Erkenntnis ist mit Nicolaus Cusanus als kreative, asymptotische Assimilation zu fassen. ${ }^{2}$ Menschliches Erkennen ist zunächst einmal Assimilation, d.h. Angleichung - bei Cusanus besser: Anähnlichung - von Erkenntnissubjekten an Erkenntnisobjekte. „[U]nser Geist ist eine verähnlichende Kraft“, ${ }^{3}$ formuliert Cusanus in ,De mente“ programmatisch. Assimilation wird, entsprechend der epistemischen Ausstattung von Menschen, auf zwei Arten vollzogen: als sinnlichrationale oder als geistige. Erstere ist mit raum-zeitlichen (d.h. sinnlich wahrnehmbaren) Erkenntnisgegenständen befasst, zweitere mit ,übersinnlichen“ - wie Ideen in ihrer cusanischen Ausprägung oder Gott. ${ }^{4}$ Cusanus beschreibt menschliches Erkennen dann genauer als asymptotische Assimilation. Während es nicht verwundert, dass er ein vollständiges Erfassen Gottes für unmöglich hält, stellt sich die Frage, warum er Erkennen grundsätzlich als asymptotische Assimilation charakterisiert. Begründet liegt das darin, dass das vollständige Durchdringen eines Erkenntnisobjekts für Cusanus das Durchdringen der Ursache dieses Objekts einschließt. Nun geht die Welt, in der sich Menschen finden und die sie

fenheit und das Tätigkeitsspektrum der mens humana diskutiert. Statt sich, wie das in anderen Werken meist der Fall ist, auf bestimmte - thematisch relevante - Aspekte der mens humana zu konzentrieren, präsentiert Cusanus in ,De mente‘ eine Art Gesamtporträt.

2 Diese Charakterisierung ist als Verdichtung epistemologischer Strukturmerkmale über die cusanischen Werke hinweg weitgehend stabil. Die einzige Einschränkung ist dahingehend zu machen, dass in ,De docta ignorantia', Cusanus' erstem philosophischen Werk, das Kreativitätsmoment höchstens implizit vorhanden ist. Als Assimilation kreativer Art bezeichnet Cusanus menschliche Erkenntnis dabei selbst; ,asymptotisch“ ist eine interpretatorische Zuschreibung. Vgl. die ausführliche Diskussion des Motivs kreativer, asymptotischer Assimilation in Christian Kny, Kreative, asymptotische Assimilation (Beiträge zur Geschichte der Philosophie und Theologie des Mittelalters, Neue Folge 84). Münster (im Druck), S. 29-101, auf der die folgende knappe Skizze dieses Motivs basiert.

3 Nicolaus Cusanus, Idiota de mente c. 7 n. 99. Hrsg. v. Renate Steiger (h ${ }^{2}$ V). Hamburg 1983: nostra mens est vis assimilativa. Die Übersetzungen aus dem Lateinischen stammen aus der zweisprachigen Meiner-Ausgabe. Wo Missverständnisse drohen, passe ich die Übersetzungen etwas an.

4 Eine ausführliche Darstellung der verschiedenen Assimilationsweisen findet sich in Cusanus, De mente (Anm. 3) c. 7 n. 100-106. 
erkennend $\mathrm{zu}$ durchdringen versuchen, auf den göttlichen Ursprung zurück. Dieser Ursprung - unendliches Maß aller Dinge, letzter Grund alles Seienden und damit das letzte Ziel allen Erkennens - ist für Menschen allerdings nicht durchdringbar. Mehr als Annäherung an den Ursprung, und damit abgeleitet an alles auf diesen Zurückgehende, ist Menschen nicht möglich. Cusanus fasst menschliches Erkennen schließlich als kreative, asymptotische Assimilation. Es ist für ihn kein irgendwie automatisch oder deterministisch ablaufender Prozess, sondern eine explizit mit dem lateinischen creare bezeichnete Tätigkeit sowohl sinnlichrationaler als auch geistiger Art. Cusanus begründet diese Kreativität mit seiner Auslegung des biblischen imago-dei-Motivs. Menschen sind dieser Auslegung zufolge keine Bilder des göttlichen Ursprungs derart, wie ein fertiges Porträt Bild eines Malers ist. Ihr zentrales Charakteristikum besteht darin, dass sie lebendige Bilder sind - sie sind vom göttlichen Ursprung nicht als unveränderliche Entitäten geschaffen, sondern mit einer gewissen Kreativität ausgestattet und der Selbstveränderung und -verbesserung fähig. Unter epistemologischen Gesichtspunkten bedeutet das für Cusanus: Menschen setzen ihre natürlichen Fähigkeiten ein, um ihr epistemisches Instrumentarium - Begriffe, Begriffszusammenhänge, Erkenntnisumgebungen - selbst hervorzubringen. Menschen vollziehen mit Cusanus Erkenntnis so als kreative, asymptotische Assimilation an ihre Erkenntnisgegenstände.

In diese grobe epistemologische Skizze können nun die eingangs angesprochenen Dimensionen von Messen eingetragen werden. Zunächst stellt Cusanus das Messen ins Zentrum von ,De mente،, wenn er mit Blick auf die titelgebende mens humana im ersten Kapitel konstatiert: „Der Geist ist das, woraus aller Dinge Grenze und Maß stammt. Mens, der Geist, wird nämlich von mensurare, messen, her benannt“. ${ }^{5}$ Weil Erkenntnistätigkeit dann im weiteren Verlauf des Werkes als die wesentliche Tätigkeit des menschlichen Geistes beschrieben wird, ${ }^{6}$ fallen ,messen' und ,erkennen‘ zusammen. Die obige Skizze menschlicher Erkenntnistätigkeit macht daher zunächst deutlich, was ,messen‘ mit Cusanus nicht ist: Es ist

5 Cusanus, De mente (Anm. 3) c. 1 n. 57: mentem esse, ex qua omnium rerum terminus et mensura. Mentem quidem a mensurando dici. Das endliche Messen von Menschen ist dabei selbst noch einmal verwiesen auf Gott als unendliches Maß alles Seienden. Vgl. etwa ebd. c. 9 n. 123 ; c. 11 n. 133.

6 Cusanus bedient sich dabei einer Gegenüberstellung von mens divina und mens humana: Erstere schafft Sein, während zweitere Sein erkennt, indem sie Begriffe schafft. So wird die mens humana einerseits an die mens divina dadurch herangerückt, dass beide mentes etwas schaffen. Andererseits wird eine klare Grenze gezogen im Blick darauf, wie und was die beiden mentes schaffen. Das (begriffliche) Schaffen der mens humana ist abhängig von und defizient gegenüber dem ursprünglichen Schaffen der mens divina. Für die beiden prominentesten Passagen vgl. Cusanus, De mente (Anm. 3) c. 3 n. 72; c. 7 n. 99. 
erstens nicht die genaue, vollständige Bestimmung von Gemessenem. Das wird durch die Charakterisierung menschlicher Erkenntnis als asymptotisch verhindert. Es ist zweitens nicht das bloße Anlegen eines menschenunabhängigen Maßes an zu Messendes. Das ist dem kreativen Moment menschlichen Erkennens geschuldet. Denn wenn Menschen erkennen, indem sie begrifflich schaffen, dann bringen sie die als Maß fungierenden Begriffe im Erkenntnisprozess erst hervor. ${ }^{7}$ Positiv gewendet heißt das: Menschen erkennen beziehungsweise messen, indem sie sich qua selbstgeschaffener Maße asymptotisch entweder von der mens divina Geschaffenem - und dazu gehören auch die Menschen selbst - oder der mens divina annähern. Menschen werden so mit ihrem erkennenden Messen in eine Weltordnung einbeschrieben, der die mens divina als absolutes Maß Sein und Gestalt verleiht.

Eine der Fragen, die Cusanus mit dieser Beschreibung menschlicher Erkenntnis provoziert, ist die nach einem Kriterium, anhand dessen erfolgreiche von erfolglosen Erkenntnisbemühungen unterschieden werden können. Damit ist die Überleitung zur zweiten hier diskutierten Dimension von Messen gemacht. Denn die Charakterisierung menschlicher Erkenntnis als kreative, asymptotische Assimilation zieht anspruchsvolle Rahmenbedingungen hinsichtlich der Kriterienfrage nach sich: Menschen können einerseits ihre epistemische Tätigkeit nicht hinreichend durch den Verweis auf Gegenstände rechtfertigen, die nicht vollständig erkennbar sind. Selbst wenn diese Gegenstände teilweise oder annäherungsweise erkennbar sind, so bleibt doch bei jeder diese Gegenstände involvierenden Behauptung eine unüberbrückbare Rechtfertigungslücke. Aussagen über einen Gegenstand können nicht von diesem Gegenstand her bewertet werden, wenn er nie ganz durchschaut werden kann. Weil nun laut Cusanus nichts vollständig erkennbar ist, das von der mens divina geschaffen ist, droht ein weitreichendes Rechtfertigungsproblem. Will Cusanus andererseits mehr als einen Kohärentismus plausibel machen, in dem Menschen nur über die von ihnen selbst geschaffenen Begriffe und Begriffsumgebungen rechtfertigbare Aussagen tätigen können, braucht er dringend ein Erkenntniskriterium.

\footnotetext{
7 Aus diesen zwei Aspekten menschlichen Messens folgt, dass die von Menschen hervorgebrachten begrifflichen Maßstäbe nicht überzeitlich stabil sind. Weil Erkenntnis als asymptotische Annäherung verläuft und das begriffliche Instrumentarium dieser Annäherung im Zuge der Annäherung erst geschaffen wird, ist mit dem Annäherungsprozess auch dessen begriffliches Instrumentarium Veränderungen unterworfen.
} 


\section{Menschen als, Einfaltungen' mit anerschaffener Urteilsfähigkeit}

Einen Erklärungsansatz bezüglich der Kriterienfrage bietet Cusanus’ Beschreibung von Menschen als ,Einfaltungen“ (complicationes) mit anerschaffener Urteilsfähigkeit (iudicium concreatum). ${ }^{8}$ Wenn Menschen als Einfaltungen das Maß bereits besitzen, anhand dessen sie Erkenntnisvorgänge mit ihrer Urteilsfähigkeit bewerten, kann das Rechtfertigungsproblem umgangen werden: Einerseits ist die Gefahr von Maßlosigkeit im Sinne des völligen Fehlens jeglichen Maßes abgewendet; andererseits können Verfehlungen des vorhandenen Maßes, etwa in Form von falschen Urteilen, als solche identifiziert und korrigiert werden.

Ich beginne mit dem iudicium concreatum, das in ,De mente“ im Rahmen einer Stellungnahme $\mathrm{zu}$ aristotelischen und platonischen Umgangsformen mit Ideen eingeführt wird. Cusanus lehnt zunächst ein platonisches Modell anerschaffener Ideen $\mathrm{ab}$, welche die Seele im Leib verloren habe und an die sie sich dann wiedererinnern müsse. Auch einem aristotelischen tabula-rasa-Modell stimmt er jedoch nicht uneingeschränkt zu. Am platonischen Modell kritisiert er, dass der Leib darin zum Hindernis degradiert werde; am aristotelischen, dass ein völlig unbeschriebenes Blatt in seinen Erkenntnisbemühungen in Halt- und damit Maßlosigkeit abrutsche. ${ }^{9}$ Als Mittelweg wird daher das iudicum concreatum eingeführt:

Weil unser Geist indessen nicht vorankommen kann, wenn ihm jedes Urteil fehlt [...], deshalb hat unser Geist eine ihm anerschaffene Urteilsfähigkeit, ohne die er keine Fortschritte machen könnte. Diese Urteilskraft ist dem Geist von Natur aus anerschaffen. Durch sie urteilt er selbständig über Beweisgründe, ob sie schwach, stark oder schlüssig sind. ${ }^{10}$

8 Was Cusanus mit complicatio und iudicium concreatum meint, wird im Folgenden erläutert. Ich lasse die Ausdrücke hier deshalb noch unkommentiert stehen.

9 Vgl. Cusanus, De mente c. 4 n. 77 (Anm. 3). Die Kritik an der Degradierung des Leibes weist auf ein christliches Spannungsfeld hin: einerseits ist Sünde eng an Körperlichkeit (Lust, Trieb, usw.) gebunden; andererseits sind Menschen von Gott als leibliche Wesen geschaffen, was eine radikale Leibfeindlichkeit problematisch macht (vgl. in diesem Kontext auch den Beitrag zu Askese in diesem Band). Letzteres kann als Grund dafür gesehen werden, warum Cusanus den Leib hier nicht als Hindernis menschlicher Erkenntnistätigkeit verstanden wissen will. Inwiefern er Platon und Aristoteles mit seinen kurzen Positionsbeschreibungen angemessen wiedergibt, spielt hier keine Rolle; wichtig ist, welche Annahmen Cusanus ablehnt - denn daran wird ersichtlich, wie seine Alternative zu verstehen ist.

10 Cusanus, De mente (Anm. 3) c. 4 n. 77: Verum quoniam non potest proficere, si omni caret iudicio [...], quare mens nostra habet sibi concreatum iudicium, sine quo proficere nequiret. Haec vis 
Cusanus stattet Menschen also mit einer Art Kompass aus, anhand dessen sie ihre Erkenntnistätigkeit bewerten können; einem Kompass, „der in unserem Geist spricht und urteilt, dies sei gut, dies gerecht, dies wahr, und uns tadelt, wenn wir vom Rechten abweichen." ${ }^{11}$ Menschen besitzen damit eine Fähigkeit, die Begriffsproduktion und begriffliche Aktivität kritisch begleitet und in die rechten Bahnen lenkt.

Zwei Präzisierungen lassen sich diesbezüglich mit ,De mente“ machen: Erstens spricht Cusanus in den zwei Passagen, in denen das iudicium concreatum auftritt, dessen Arbeitsbereich an. In der gerade zitierten Passage ist von Elementen diskursiven Denkens - rationalen Gebilden, Schlussfolgerungen - die Rede; etwas später wird das iudicium im Kontext der Erschließung von Raum-Zeitlichem (de exterioribus) eingesetzt. ${ }^{12}$ Diese beiden Passagen machen deutlich, dass das iudicium concreatum Erkenntnistätigkeit im sinnlich-rationalen Bereich bewertet. Das wirft die Frage auf, ob Cusanus dann nicht vor dem Problem steht, nur für einen Teil menschlicher Erkenntnistätigkeit ein Erkenntniskriterium formuliert zu haben - er spricht ja nicht nur von einem sinnlich-rationalen, sondern auch von einem geistigen Bereich menschlicher Erkenntnis.

Zumindest einen Antwortansatz liefert in dieser Hinsicht die zweite Präzisierung, die mit ,De mente' möglich ist. Direkt vor der Passage, in der er das iudicium concreatum mit Urteilen über Raum-Zeitliches verbindet, spricht Cusanus das Ineinandergreifen verschiedener Erkenntnisbereiche oder -formen an:

Wie der Gesichtssinn sieht und ohne die Unterscheidungskraft, die ihn unterrichtet und erleuchtet und vollendet, nicht weiß, was er sieht, so zieht der Verstand Schlüsse und weiß ohne den Geist nicht, was er schließt, sondern der Geist unterrichtet, erleuchtet und vollendet das schlussfolgernde Denken, so dass es weiß, was es schließt [...]. So ist der Geist die

iudicaria est menti naturaliter concreata, per quam iudicat per se de rationibus, an sint debiles, fortes aut concludentes.

11 Ebd. c. 4 n. 78: in mente nostra loquentem et iudicantem hoc esse bonum, hoc iustum, hoc verum, et nos reprehendentem, si declinamus a iusto. Cusanus weist dem iudicium concreatum mit dessen Aufsicht über „gut“, „gerecht“ und „wahr“ also eine Steuerungsfunktion sowohl unter theoretischen als auch unter praktischen Gesichtspunkten zu. Ich beschränke mich hier auf die theoretischen Aspekte; unter praktischen Gesichtspunkten wird das iudicium concreatum in Isabelle Mandrella, Viva imago. Die praktische Philosophie des Nicolaus Cusanus (Buchreihe der Cusanus-Gesellschaft 19). Münster 2012, bes. S. 100-103 (vgl. das Sachregister ebd., S. 324, für eine vollständige Liste der relevanten Passagen) diskutiert. Vgl. auch Klaus Kremer, Das kognitive und affektive Apriori bei der Erfassung des Sittlichen. In: Ders. (Hg.), Praegustatio naturalis sapientiae. Gott suchen mit Nikolaus von Kues (Buchreihe der Cusanus-Gesellschaft. Sonderbeitrag). Münster 2004, S. 103-146, hier S. 136-140.

12 Vgl. Cusanus, De mente (Anm. 3) c. 5 n. 85. 
unterscheidende Form der rationalen Gehalte, wie die Ratio unterscheidende Form der Sinneswahrnehmung und Vorstellungen ist. ${ }^{13}$

Bringt man diese Beschreibung mit der Liste der epistemischen Fähigkeiten zusammen, die Cusanus in ,De mente ${ }^{\prime}$ Menschen zuschreibt und als hierarchische Ordnung von Assimilationsstufen expliziert, ${ }^{14}$ legt das die Integration des auf Raum-Zeitliches gerichteten iudicium concreatum in eine Art allgemeine Beurteilungsfigur nahe: Bei ,aneinander liegenden Assimilationsformen wird jeweils von der höheren aus die niedrigere bewertet, und das iudicium concreatum ist für eine dieser Bewertungsstufen zuständig. ${ }^{15}$

Weil Cusanus nun die Beurteilung von Raum-Zeitlichem als das Schauen von Menschen auf etwas beschreibt, das in ihnen selbst liegt, ${ }^{16}$ drängt sich die Frage auf: Worauf wird da geschaut? Das leitet über zum zweiten Aspekt, der hier neben dem iudicium concreatum anzusprechen ist - der Beschreibung von Menschen als ,Einfaltungen‘. Die Figur von ,Einfaltung' und ,Ausfaltung'/,Entfaltung' (complicatio und explicatio) verwendet Cusanus oft und in verschiedenen, ontologischen wie epistemologischen Kontexten. ${ }^{17}$ In ihren Grundzügen bringt die Figur ein

13 Ebd. c. 5 n. 84: sicut visus videt et nescit quid videat sine discretione, quae ipsum informat et dilucidat et perficit, sic ratio syllogizat et nescit quid syllogizet sine mente, sed mens informat, dilucidat et perficit ratiocinationem, ut sciat quod syllogizet [...]. Ita mens est forma discretiva rationum sicut ratio forma discretiva sensuum et imaginationum. Cusanus ist in dieser Passage, wie das allgemein für ihn kennzeichnend ist, terminologisch flexibel - er verwendet Begriffe in teils unmittelbar aufeinander folgenden Passagen mit unterschiedlichen Umfängen. So kann ,Geist ${ }^{\star}$ (mens), hier für geistige im Unterschied zu sinnlich-rationaler Aktivität verwendet, auch für das Gesamt menschlicher Fähigkeiten und Aktivitäten stehen - vgl. etwa Cusanus, De mente (Anm. 3) c. 1 n. 57; c. 5 n. 80. Schlüsse vom Vorkommen eines Begriffs in einer Passage auf das Vorkommen eines bestimmten Theorems in dieser Passage sind deshalb interpretatorisch problematisch; die jeweiligen Textumgebungen müssen berücksichtigt werden.

14 Vgl. Cusanus, De mente (Anm. 3) c. 5 n. 80; c. 7 n. 100-106.

15 Am oberen Ende des Spektrums hinterlässt das allerdings eine Bewertungslücke - die höchste Form menschlicher Erkenntnistätigkeit kann als solche von Menschen selbst nicht mehr bewertet werden, da kein übergeordneter Standpunkt eingenommen werden kann. Für eine erschöpfende Antwort auf die Frage nach dieser Beurteilungsfigur müssten die verschiedenen Assimilationsformen genauer untersucht und in ihrem - nicht immer unproblematischen - Verhältnis zueinander beleuchtet werden. Das kann ich hier aus Umfangsgründen nicht leisten. Ich beschränke mich deshalb auf die Bewertung rationaler Erkenntnisvorgänge mittels des iudicium concreatum. Vgl. auch Nicolaus Cusanus, De coniecturis I c. 10 n. 52. Hrsg. v. Karl Bormann, Josef Koch u. Hans G. Senger (h III). Hamburg 1972, wo eine umfassendere Beurteilungsfigur in der angesprochenen Gestalt formuliert wird.

16 Vgl. Cusanus, De mente (Anm. 3) c. 5 n. 85.

17 Vgl. Arne Moritz, Explizite Komplikationen. Der radikale Holismus des Nikolaus von Kues (Buchreihe der Cusanus-Gesellschaft 14). Münster 2006 für eine ausführliche Studie zum Thema 
bestimmtes Verhältnis von Einheitlichkeit und Vielheitlichkeit zum Ausdruck: Eine complicatio ist einheitlich und - nicht formal-inhaltsleer, sondern als (Merkmals-) Fülle - allgemein, was explicationes auf je bestimmte Weise ausschnitthaft konkretisieren. Die Pflanze auf meinem Schreibtisch ist ein bestimmter Drachenbaum. Sie kann nicht alle Merkmale, die Drachenbäume haben können, vollumfänglich ausprägen, sondern unterscheidet sich von anderen bestimmten Drachenbäumen. Alle bestimmten Drachenbäume sind aber explicationes der complicatio ,Drachenbaum‘ und stehen als solche in einem Abhängigkeitsverhältnis zu dieser.

Menschen werden von Cusanus in ,De mente“ als besondere complicationes charakterisiert:

Alles ist in Gott, aber dort als Urbilder der Dinge; alles ist in unserem Geist, aber dort als Ähnlichkeiten der Dinge. Wie Gott die absolute Seinsheit ist, die aller Seienden Einfaltung ist, so ist unser Geist jener unendlichen Seinsheit Bild, das aller Abbilder Einfaltung ist. ${ }^{18}$

Der Abstand zwischen göttlicher complicatio und menschlichen complicationes wird von Cusanus zwar konsequent aufrechterhalten - Menschen sind sekundäre complicationes, Gott ist die primäre oder absolute complicatio. Menschen zeichnen sich aber dadurch aus, dass sie in einem ähnlichen Verhältnis zu ,ihren“ explicationes stehen wie der göttliche Ursprung zu ,seinen‘.

Zwei Aspekte dessen, was menschliche complicationes ausmacht, stehen in ,De mente“ im Vordergrund. Sie sind erstens begriffliche und zweitens lebendige Einfaltungen. Darin, dass sie Einfaltungen von Begrifflichem im Gegensatz zu Seiendem sind, besteht der maßgebliche Unterschied zwischen Menschen und dem absoluten Ursprung: Menschen falten Begriffe von dem ein, was ist, und erkennen so Gegebenes - im Unterschied zum absoluten Ursprung, in dem ,Seiendes erkennen “ und ,Seiendes schaffen“ zusammenfallen. ${ }^{19}$ Darin, dass Menschen lebendige Einfaltungen sind, besteht die maßgebliche Parallele zwischen Menschen und dem göttlichen Ursprung. Denn im Unterschied $\mathrm{zu}$ anderen, statischen Bildern dieses Ursprungs sind sie wie dieser mit Schaffenskraft aus-

mit ontologischem Schwerpunkt. Ebd., S. 76-90 wird ,De mente‘ diskutiert. Vgl. auch Klaus Reinhardt, Complicatio - explicatio. In: Cecilia Rusconi (Hg.), Manuductiones. Festschrift zu Ehren von Jorge M. Machetta und Claudia D’Amico (Texte und Studien zur europäischen Geistesgeschichte 8). Münster 2014, S. 81-91; Thomas Leinkauf, Nicolaus Cusanus. Eine Einführung (Buchreihe der Cusanus-Gesellschaft 15). Münster 2006, hier S. 102-110.

18 Cusanus, De mente (Anm. 3) c. 3 n. 73: Omnia in deo sunt, sed ibi rerum exemplaria; omnia in nostra mente, sed ibi rerum similitudines. Sicut deus est entitas absoluta, quae est omnium entium complicatio, sic mens nostra est illius entitatis infinitae imago, quae est omnium imaginum complicatio.

19 Vgl. ebd.c. 3 n. 72 ; c. 4 n. 74; c. 5 n. 81; c. 7 n. 99. 
gestattet. Sie können sich selbst verbessern, sie können sich auf sich selbst beziehen und sie leben ihre Schaffenskraft vorwiegend begrifflich aus. ${ }^{20}$

Als Einfaltungen tragen Menschen das in sich, anhand dessen das iudicium concreatum rationale Erkenntnistätigkeit bewertet.

Er [sc. der menschliche Geist] hat sie [sc. die Urteilsfähigkeit] daher, weil er das Bild des Urbildes von allem ist. Gott ist nämlich das Urbild von allem. Da das Urbild von allem im Geist wie die Wahrheit im Abbild widerstrahlt, so hat er das in sich, auf das er schaut und nach dem er ein Urteil über das Außenliegende fällt. ${ }^{21}$

Weil sie lebendige Bilder Gottes sind und als solche die Urbilder der Dinge abbildhaft in sich eingefaltet finden, können Menschen die Produkte rationaler Erkenntnistätigkeit auf ihre Angemessenheit hin bewerten, indem sie auf das schauen, was sie einfalten.

Cusanus kann also nicht vorgeworfen werden, dass er die Kriterienfrage ignorierte; er diskutiert sie zwar nicht systematisch oder ausführlich, nimmt mit der Beschreibung von Menschen als complicationes mit einem iudicium concreatum aber durchaus zur Thematik Stellung. Zu fragen ist allerdings, wie befriedigend diese Stellungnahme ist. Denn Cusanus geht nicht ins Detail, so dass es bei genauerem Hinsehen bisweilen mehr Fragen als Antworten gibt.

20 Zusätzlich zu den in der vorangehenden Anmerkung genannten Stellen vgl. hier v.a. die Metapher vom lebendigen Bild in Cusanus, De mente (Anm. 3) c. 13 n. 149. Ich spare diese Metapher hier aus, weil sie in der Forschung ausführlich diskutiert ist. Vgl. unter verschiedenen Gesichtspunkten und mit weiteren Literaturverweisen Isabelle Mandrella, Viva imago. Der Einfluss des Raimundus Sabundus auf die cusanische Metapher der viva imago. In: Rusconi (Anm. 17), S. 223-241; Mandrella (Anm. 10), S. 211-223, 253-263, 271-287; Anke Eisenkopf, Das Bild des Bildes. Zum Begriff des toten und lebendigen Bildes in Idiota de mente. In: Inigo Bocken u. Harald Schwaetzer (Hgg.), Spiegel und Porträt. Zur Bedeutung zweier zentraler Bilder im Denken des Nicolaus Cusanus. Festgabe für Klaus Reinhardt zum 70. Geburtstag. Maastricht 2005, S. 49-73; Gerda von Bredow, Der Geist als lebendiges Bild Gottes (Mens viva imago dei). In: Dies., Im Gespräch mit Nikolaus von Kues. Gesammelte Aufsätze 1948-1993. Hrsg. v. Hermann Schnarr (Buchreihe der Cusanus-Gesellschaft. Sonderbeitrag). Münster 1995, S. 99-109.

21 Cusanus, De mente (Anm. 3) c. 5 n. 85: Habet ex eo, quia est imago exemplaris omnium. Deus enim est omnium exemplar. Unde cum omnium exemplar in mente ut veritas in imagine reluceat, in se habet ad quod respicit, secundum quod iudicium de exterioribus facit. Es ist in der epistemologischen Tradition üblich, der sinnlichen ,Außenschau' eine intellektuelle ,Innenschau' zu parallelisieren. Cusanus verwendet hier respicere; intueri ist ein anderer gebräuchlicher Ausdruck in diesem Kontext. Die genannte Parallelisierung hebt nicht darauf ab, dass man intellektuell Erfassbares tatsächlich sehen könne. Im Kern steht vielmehr Unmittelbarkeit: Die übliche Charakterisierung intellektuellen Erfassens als unmittelbar wird metaphorisch begleitet durch die Begrifflichkeit - als unmittelbar erfahrener - visueller Wahrnehmung. 


\section{Was heißt ,einfalten'?}

Die Frage, auf die ich mich hier konzentriere, lautet: Was genau ist darunter zu verstehen, dass Menschen lebendige complicationes sind? Was also falten Menschen ein? Im Blick worauf bewertet das iudicium concreatum rationale Erkenntnistätigkeit?

Cusanus selbst spricht in ,De mente - bei der Einführung des iudicium concreatum und an anderen Stellen - üblicherweise drei Theorieoptionen an: eine platonische, eine aristotelische und einen (meist harmonisierenden) Mittelweg. ${ }^{22}$ Das ist im Rahmen interpretatorischer Zugriffe zu berücksichtigen - expliziert man Cusanus' ,Mittelwege' einseitig platonisch oder aristotelisch, dann muss erklärt werden, wie das mit seiner eigenen Positionierung kompatibel ist.

Im Rahmen der Frage danach, was unter menschlichen Einfaltungen genau zu verstehen ist, gibt es bestimmte Passagen in ,De mente', denen sich jeder Interpretationsansatz stellen muss. Deshalb liste ich zunächst repräsentative Stellen auf, bevor ich die verschiedenen interpretatorischen Optionen diskutiere.

(I) Alles ist in Gott, aber dort als Urbilder der Dinge; alles ist in unserem Geist, aber dort als Ähnlichkeiten der Dinge. Wie Gott die absolute Seinsheit ist, die aller Seienden Einfaltung ist, so ist unser Geist jener unendlichen Seinsheit Bild, das aller Abbilder Einfaltung ist.23

(II) Weil nun der Geist ein gewisser göttlicher Same ist, der mit seiner Kraft aller Dinge Urbilder begrifflich einfaltet, ist er von Gott, von dem er diese Kraft hat [...], zugleich in den passenden Boden gesetzt worden, wo er Frucht bringen und aus sich die Gesamtheit der Dinge begrifflich ausfalten kann..$^{24}$

(III) Darin scheint also Aristoteles der richtigen Meinung zu sein, dass der Seele keine Begriffe anerschaffen sind, die sie beim Eintritt in den Leib verloren hätte. ${ }^{25}$

(IV) Er [sc. der menschliche Geist, $c k$ ] hat sie [sc. die Urteilskraft] daher, weil er das Bild des Urbildes von allem ist. Gott ist nämlich das Urbild von allem. Da das Urbild von allem im Geist wie die Wahrheit im Abbild widerstrahlt, so hat er das in sich, auf das er schaut und nach dem er ein Urteil über das Außenliegende fällt [...]. <Das ist so,> wie wenn die einfachste und unteilbare Spitze eines Winkels an einem ganz fein geschliffenen Diamanten, in der die Formen aller Dinge widerstrahlten, lebendig wäre, dann würde jene, wenn sie

22 Solche ,Mittelwege' finden sich neben den Passagen zum iudicium concreatum etwa in Cusanus, De mente (Anm. 3) c. 12 n. 142-144; c. 14 n. 152f.

23 Anm. 17.

24 Cusanus, De mente (Anm. 3) c. 5 n. 81: Unde quia mens est quoddam divinum semen sua vi complicans omnium rerum exemplaria notionaliter, tunc a deo, a quo hanc vim habet [...], est simul et in convenienti terra locatum, ubi fructum facere possit et ex se rerum universitatem notionaliter explicare.

25 Ebd. c. 4 n. 77: In hoc igitur Aristoteles videtur bene opinari animae non esse notiones ab initio concreatas, quas incorporando perdiderit. 
sich anblickte, aller Dinge Abbilder finden, durch die sie sich Begriffe von allem machen könnte. ${ }^{26}$

(V) Und in dieser Assimilation [sc. der geistigen Assimilation an reine Formen, $c k$ ] verhält sich der Geist, wie wenn die Bildsamkeit losgelöst von [...] allen bildsamen Stoffen lebendig wäre in geistigem Leben, so dass sie sich durch sich selbst allen Gestalten, wie sie in sich und nicht in der Materie bestehen, verähnlichen könnte. Eine solche würde nämlich gewahr werden, dass in der Kraft ihrer lebendigen Bildsamkeit, d.h. in ihr selbst, die Begriffe von allem enthalten sind, weil sie sich allem angleichen könnte. ${ }^{27}$

(I) und (II) sind programmatische Passagen, in denen Cusanus den Einfaltungscharakter von Menschen und deren Verhältnis zur göttlichen complicatio anspricht. (III) ist die bereits diskutierte Passage, in der Cusanus das iudicicum concreatum als Mittelweg zwischen anerschaffenen Begriffen und tabula rasa einführt. (IV) verknüpft das iudicium mit der Beschreibung von Menschen als complicationes, während (V) beschreibt, wie sich der menschliche Geist im Rahmen seiner Assimilationstätigkeit selbst als ,Ort‘ aller Begriffe erfasst.

Diese Stellensammlung zeigt die Grundspannung, mit der Interpretationsversuche im Blick darauf, was Menschen einfalten, konfrontiert sind. Einerseits lehnt Cusanus anerschaffene Begriffe ab. Andererseits bezeichnet er Menschen als Einfaltungen der Begriffe von allem, die beim Einsatz ihres iudicium auf das blicken, was sie einfalten. Wenn Menschen nun ihre rationale Erkenntnistätigkeit beurteilen, indem sie auf Begriffliches blicken, das sie einfalten - müssen dann nicht Begriffe, die ihnen laut (III) nicht anerschaffen sind, bei konkreten Erkenntnisvorgängen bereits als Orientierungsgrößen vorhanden sein? Wie könnte die Zuverlässigkeit dieser Orientierungsgrößen gewährleistet werden, wenn bei ihrem Erwerb selbst noch keine Orientierungsgrößen vorhanden sind? Kurz: Der Umgang mit (III) ist ein interpretatorisches Problem.

Zwei Interpretationslinien gibt es diesbezüglich, die jeweils in einer ,harten“ und einer ,weichen' Variante ausgeprägt werden können: Option (1a) besteht darin, (III) und die zugehörige Textumgebung schlicht nicht zu berücksichtigen und Menschen doch anerschaffene Begriffe zuzuschreiben. Option (1b) erkennt

26 Ebd. c. 5 n. 85: Habet ex eo, quia est imago exemplaris omnium. Deus enim est omnium exemplar. Unde cum omnium exemplar in mente ut veritas in imagine reluceat, in se habet ad quod respicit, secundum quod iudicium de exterioribus facit [...]. Ac si acuties simplicissima et indivisibilis anguli lapidis diamantis politissimi, in qua omnium rerum formae resplenderent, viva foret, illa se intuendo omnium rerum similitudines reperiret, per quas de omnibus notiones facere posset.

27 Ebd. c. 7 n. 104: Et in hac assimilatione se habet mens, ac si flexibilitas absoluta a [...] omnibus flexibilibus foret viva vita mentali, ut ipsa per se ipsam omnibus figuris, ut in se et non in materia subsistunt, assimilare possit. Talis enim in vi suae flexibilitatis vivae, hoc est in se, notiones omnium, quoniam omnibus se conformare posset, esse conspiceret. 
(III) samt Textumgebung zwar an, schafft durch eine Ausdifferenzierung aber Raum für Anerschaffenes, im Blick auf das Menschen ihr iudicium einsetzen können. Option (2a) nimmt (III) beim Wort und erklärt Menschen, abgesehen vom iudicicum concreatum, als völlig ,leer; jegliche Gehalte müssen erst durch Abstraktion ,von außen' erworben werden. Option (2b) nimmt (III) ernst, erlaubt aber das Hervorbringen von Gehalten ,von innen heraus‘.

Als unplausibel ausgeschlossen werden können zunächst einmal die ,harten` Optionen (1a) und (2a). Nicht nur repräsentieren sie die Art von Platonismus und Aristotelismus, die Cusanus in der Umgebung von (III) und andernorts ${ }^{28}$ explizit ablehnt. Sie kollidieren auch jeweils mit zentralen Annahmen, die Cusanus in ,De mente“ vertritt. Mit Blick auf (1a) ist das vor allem die Beschreibung von Menschen als Bilder der göttlichen Schaffenskraft: ${ }^{29}$ Wenn Menschen Begriffe bereits als fertige anerschaffen sind, dann müssen sie diese Begriffe nicht erst hervorbringen, sondern nur wiederfinden. Der Bildcharakter von Menschen liefe damit in einem wesentlichen Aspekt ins Leere, was angesichts der Bedeutung dieses Aspekts interpretatorisch nicht tragbar ist. Von den Schwierigkeiten, mit denen (2a) konfrontiert ist, seien hier zwei genannt. Einerseits versteht Cusanus eine complicatio als dem Seinsgehalt nach reicher im Vergleich mit ihren explicationes. Ein leerer Geist müsste gemäß (2a) also dem Seinsgehalt nach reicher sein als die begrifflichen Gehalte, die er erwirbt. Andererseits beschreibt Cusanus - etwa in (II) und (V) - assimilierendes Entfalten als ein Entfalten ,von innen heraus'. Wenn Gehalte aber ,von außen` erworben werden, dann werden sie gerade nicht ,von innen heraus' entfaltet.

(1b) operiert mit einer Unterscheidung zwischen - aktual verstandenen Begriffen und nicht-begrifflichen Gehalten. Das schafft Raum dafür, (III) gelten zu lassen und dennoch Gehalte anzusetzen, auf die sich Menschen mit ihrem iudicium beziehen können - ohne, dass sie diese Gehalte erst ,von außen“ erwerben müssten. Ein prominenter Vertreter dieser Linie ist Klaus KREMER:

Nicht fertige Ideen bzw. Begriffe haben wir a priori in uns, wohl aber ein gleichsam inhaltliches Material [...], durch das wir die Begriffe zur Beurteilung des in der Sinneserfahrung Begegnenden bilden. ${ }^{30}$

Die Vorteile dieser Interpretationslinie bestehen darin, dass sie erstens keine Schwierigkeiten hat, die Seinsfülle der complicatio $\mathrm{zu}$ erklären. Menschen sind

28 Vgl. unter verschiedenen Gesichtspunkten Cusanus, De mente (Anm. 3) c. 2 n. 65-67; c. 5 n. 142-144; c. 12 n. 80 ; c. 13 n. 145 f.

29 Am prägnantesten findet sich diese Beschreibung in der Metapher vom lebendigen Bild. Vgl. Anm. 20.

30 Kremer (Anm. 11), S. 109. Vgl. ebd., S. 107-115 für den ganzen hier relevanten Textabschnitt. 
zwar nicht mit anerschaffenen Begriffen ausgestattet, aber doch mit etwas, auf das geblickt und aus dem heraus entfaltet werden kann. Deshalb ist zweitens auch das Abhängigkeitsverhältnis zwischen explicationes und complicatio plausibel. Aus dem, was Menschen implizit in sich tragen, können sie eine Vielheit von explicationes begrifflich entfalten, welche die Seinsfülle ,mens ${ }^{\star}$ auf je spezifische Weise konkretisieren, ohne sie je vollständig ausprägen zu können. Drittens gibt es auch für die Kreativitätsthese Raum - Cusanus bezeichnet die Kreativität von Menschen als begriffliche, so dass die Verbegrifflichung impliziter Gehalte als Ausdruck dieser Kreativität interpretiert werden kann. Die verschiedenen Gesichtspunkte von complicatio, iudicium concreatum und deren Verhältnis zueinander, die in (I)-(V) zum Ausdruck gebracht sind, erscheinen kompatibel.

Diese Interpretationslinie sieht sich jedoch auch kritischen Anfragen ausgesetzt. Man kann erstens fragen, was genau die impliziten Gehalte - das ,gleichsam inhaltliche Material“, in Kremers Worten - sein sollen, die postuliert werden. Cusanus zufolge faltet eine complicatio alles ein, was aus ihr entfaltet sein oder entfaltet werden kann. Nachdem Menschen gemäß (I), (II), (IV) und (V) alles begrifflich entfalten können, müssen sie alles in Form einer nicht-begrifflichen ,Materialsammlung‘ einfalten. Das klingt verdächtig nach der Behauptung eines Phantoms - ,alles' soll implizit vorhanden sein, ohne dass klar wäre oder klar gemacht würde, wie das gemeint ist. Selbst wenn die Textbasis bei Cusanus als unzureichend für eine eindeutige Antwort angesehen wird, muss eine zufriedenstellende Interpretation zumindest Antwortmöglichkeiten anbieten können. Man kann in (1b) zweitens die Integration der von Cusanus betonten Kreativität und Dynamik menschlicher Erkenntnistätigkeit als bloßes Lippenbekenntnis kritisieren. Wenn er etwa in (II) davon spricht, dass der menschliche Geist in seiner oder durch seine Kraft alles begrifflich einfalte, dann wird damit nicht nur ent- sondern auch einfalten als Tätigkeit oder Aktivität beschrieben. Wenn der Geist im Rahmen von (1b) dagegen als ,Materialsammlung' beschrieben wird, dann legt das ein statisches Eingefaltet-Sein von Gehalten nahe und beschränkt Aktivität und Kreativität auf die entfaltende Tätigkeit von Menschen, deren Kreativitätsgrad fraglich ist. Angesichts des Aufwands, den Cusanus in ,De mente 'im Sinne einer Dynamisierung menschlicher Erkenntnisprozesse betreibt, ist das ein ernstzunehmender Vorwurf. (1b) zeigt sich damit als plausible, aber nicht unproblematische Interpretationslinie.

(2b) legt den Fokus gerade auf den dynamisch-kreativen Charakter, den Cusanus menschlichen complicationes zuspricht. Diese Interpretationslinie fasst complicationes nicht als Einfaltungen im Sinne einer vorgängigen oder primären ,Materialsammlung،, auf die dann in einem Folgeschritt entfaltend zugegriffen würde. Stattdessen beschreibt sie sowohl rationale Erkenntnistätigkeit als auch deren ideelles ,Anschauungsmaterial` als zwei Momente eines menschlichen Ein- 
und Entfaltungsprozesses, in dem beides, Rationales wie Ideelles, erst hervorgebracht wird. In dieser Hervorbringung manifestiert sich eine Kreativität in emphatischem Sinne, die weder vorgängiger Gehalte in der mens humana noch von außen zugetragener Gehalte bedarf. Nimmt man ernst, dass Cusanus in ,De mente` Menschen als lebendige Bilder der göttlichen Schaffenskraft beschreibt, ${ }^{31}$ dann kann man die These vertreten: Menschen müssen Ideen oder implizite Gehalte nicht bereits (implizit) in sich tragen, um aus ihnen in einem Folgeschritt etwas entfalten zu können. Als lebendige Bilder sind sie gerade dadurch ausgezeichnet, dass sie in einem komplementären Prozess Ideen bzw. Einfaltungen und ihre begrifflichen Entfaltungen, d.h. Maß und Gemessenes schaffen. Indem ich begrifflich auf diesen Drachenbaum zugreife - ihn benenne, ihm Merkmale zuschreibe, ihn von den anderen Zimmerpflanzen im Raum abgrenze -, bringe ich gleichzeitig den ideellen Rahmen davon mit hervor, was Drachenbäume sind. Indem ich auf den ideellen Rahmen ,Drachenbaum' blicke, binde ich meine Schritte im begrifflichen Zugriff auf diesen Drachenbaum an den ideellen Rahmen als Maß zurück. Kurz: Im Ausfalten falte ich ein, im Einfalten falte ich aus. (2b) unterscheidet sich damit von (2a) nicht dadurch, dass neben dem iudicium concreatum doch anerschaffene Gehalte angesetzt würden. Der Unterschied besteht vielmehr darin, dass die tabula rasa nicht durch Abstraktion von außen, sondern durch Hervorbringung von innen ,beschriftet' wird.

Arne MoRitz stellt durch seine Darstellung von complicatio und explicatio wesentliche Teile des Fundaments dieser Interpretationslinie zur Verfügung. Auf der Basis einer Liste von Merkmalen, die er anhand dreier cusanischer Texte entwickelt, ${ }^{32}$ kritisiert er gängige Interpretationsmuster:

Es schien so als sei mit der Rede von der mens als imago complicationis dei ein ,Wesen des Geistes‘ gemeint, eine ,Ursprungseinheit‘, in der die ,Erkenntnisinhalte schon implizit enthalten sind', woraus sie im explikativen Prozess hervorgebracht werden. ,Die Analogie zwischen dem göttlichen und dem menschlichen Geist‘ schien nur darin zu bestehen, ,dass beide die Totalität des von ihnen Hervorgebrachten schon in sich enthalten und dass beide der aktive Ursprung dessen sind, was aus dieser Totalität hervorgeht.' Auch bei der mens schien der Gedanke eine vorgängige ,Potentialität der Komplikation' und die ,Aktualität ihrer Explikation' zu bezeichnen. ${ }^{33}$

31 Vgl. Anm. 20.

32 Vgl. Moritz (Anm. 17), S. 27-90 (bei den Texten handelt es sich um ,De docta ignorantia،, einen Brief an Rodrigo Sanchez de Arévalo aus dem Jahr 1442 und ,De mente`). Die Merkmalliste findet sich ebd., S. 120-155.

33 Ebd., S. 244f. Die von Moritz zitierten Passagen stammen - vgl. ebd., Anm. 727-729 für die Seitenangaben - aus Theo van Velthoven, Gottesschau und menschliche Kreativität. Studien zur Erkenntnislehre des Nikolaus von Kues. Leiden 1977 und Tilman Borsche, Was etwas ist. Fragen nach der Wahrheit der Bedeutung bei Platon, Augustin, Nikolaus von Kues und Nietzsche. 
Versteht man mit Moritz den menschlichen Geist als etwas, das sich in Entfaltungsprozessen zu Gehalten sinnlich-rationaler wie geistiger Art verdichtet und sich so als complicatio realisiert, dann sind (I)-(V) ebenfalls kompatibel. Denn Cusanus behauptet nirgends, dass Menschen Gehalte, seien sie nun expliziter oder impliziter Art, in irgendeiner Form vorgängig bereits mitbringen. Zusätzlich zu der Tatsache, dass (2b) den cusanischen Kreativitätsgedanken stark machen kann, sieht sich die Interpretationslinie gar nicht erst mit der Notwendigkeit konfrontiert, mit vorgängigen impliziten Gehalten ein epistemologisches ,Phantom" postulieren zu müssen.

Auch (2b) ist allerdings kritisch zu befragen. Vorwürfen, Menschen würden hier mit zu weitreichenden Schaffenskräften ausgestattet, muss man nicht begegnen. Denn solange Menschen nur epistemisch und nicht ontisch schaffen, ist das für Cusanus kein Problem, sondern gerade Ausdruck der Darstellung der mens humana als Bild der göttlichen Schaffenskraft. Ernstzunehmen ist dagegen der Einwand, (2b) vernachlässige die Abhängigkeit der explicationes von ihrer jeweiligen complicatio, wenn - was die Interpretationslinie zu ermöglichen scheint Sinnlich-Rationales hervorgebracht wird ohne die zugehörige geistige complicatio; etwa eine Behauptung über diesen Drachenbaum auf meinem Schreibtisch, ohne dass ,Drachenbaum“ als Orientierungsgröße hervorgebracht wäre. Ebenfalls ernstzunehmen ist schließlich der Vorwurf, (2b) könne die Seinsfülle der complicatio ,Mensch“ nicht plausibel machen. Denn wenn sinnlich-rationale wie geistige Hervorbringungen ohne Rückgriff auf bereits Vorhandenes hervorgebracht werden, scheint die Charakterisierung der mens humana als komplikative Seinsfülle ebenso phantomhaft wie das Ansetzen eines nicht-begrifflichen ,Alles‘.

Sowohl von (1b) als auch von (2b) aus kann den je angeführten Kritikpunkten dabei durchaus begegnet werden. Aus Umfangsgründen kann ich das hier allerdings nicht unternehmen. Ich belasse es deshalb bei einigen abschließenden Hinweisen. Erstens ist festzuhalten, dass auch die plausibleren Interpretationsversuche hinsichtlich der Frage, was eine menschliche complicatio genau einfalte und woran sich ihr iudicium orientiere, mit substantiellen Schwierigkeiten konfrontiert sind. Cusanus äußert sich nicht klar genug für eindeutige Urteile oder gänzlich zufriedenstellende Antworten, so dass Interpretationsansätze hinsichtlich der hier diskutierten Fragen bis zu einem gewissen Grad genau das bleiben müssen: Ansätze. Es ist zwar unbestreitbar, dass Messen für Cusanus eine zen-

München 1990. Die Linie, von der Moritz sich abgrenzt, ist in der Forschung dominant. Vgl. neben den in Moritz (Anm. 17), S. 239-251 genannten Vertreterinnen und Vertretern auch Leinkauf (Anm. 17), S. 102-110 und Reinhardt (Anm. 17). 
trale epistemologische Rolle spielt. Wie dieses Messen genau zu verstehen ist, bleibt unter den hier diskutierten Gesichtspunkten aber fraglich. Zweitens scheint mir angesichts von ,De mente‘ eine dynamische Interpretation menschlicher Erkenntnistätigkeit und damit verbunden dessen, was unter einer complicatio zu verstehen ist, sinnvoll. Cusanus legt zu viel Wert auf den Kraft- und Tätigkeitscharakter der mens humana, als dass statische Interpretationsmodelle dem Text gerecht würden. Das legt nahe, sich der Problemlage eher von (2b) aus zu nähern. Man könnte (1b) - das wäre Teil der Strategie, den Einwänden entgegenzutreten zwar dynamischer ausdeuten; (1b) bedient aber tendenziell Interpretationsreflexe neuplatonischer Provenienz, die zu einer Eingliederung von Cusanus in die entsprechende Tradition ohne unvoreingenommenes Durchdenken seiner Aussagen führen können. Um ,De mente' in seiner Eigenheit ernstzunehmen, halte ich (2b) interpretationsstrategisch deshalb für den besseren Ausgangspunkt.

\section{Schluss}

Kann Cusanus der epistemologischen Maßlosigkeit entgehen, die ihm angesichts eines möglicherweise unzureichenden Kriteriums menschlicher Erkenntnis droht? Die Antwort auf diese Frage fällt, wie deutlich wurde, zwiespältig aus. Cusanus erliegt nicht epistemologischer Maßlosigkeit in dem Sinne, dass er die Frage nach einem Kriterium menschlichen Erkennens - Messens, in seiner Terminologie - entweder ignorierte oder grundsätzlich nicht beantworten könnte. Indem er Menschen in ,De mente' mit einem anerschaffenen iudicium ausstattet, das sinnlich-rationale Erkenntnisvorgänge mit Blick auf das bewertet, was Menschen als lebendige Bilder der göttlichen Schaffenskraft einfalten, sichert er diese sinnlich-rationalen Vorgänge kriteriell ab. Dass er dabei weder besonders detailscharf vorgeht noch die Fragen, die diese Absicherung aufwirft, diskutiert, stellt interpretatorische Zugriffsversuche allerdings vor Probleme. Die beiden Zugriffsvarianten, die hier diskutiert wurden, ringen mit derselben Frage: Was genau ist es, das Menschen einfalten? Tragen Menschen implizit Gehalte in sich, die dann begrifflich entfaltet werden können? Oder realisieren Menschen gerade dadurch ihr Potential als complicationes, dass sie - ohne das Vorhandensein impliziter Gehalte - kreativ explicationes hervorbringen?

Mehr als eine Erläuterung des Problems und Ansätze zum Umgang damit kann ich hier nicht liefern. Aufbauend auf die erzielten Resultate bestünden die nächsten Schritte nun darin, die plausiblen Interpretationsvarianten zunächst eingehender zu prüfen und dann über ,De mente' hinaus ihre Kompatibilität mit den cusanischen Texten zu untersuchen. 